\title{
Experience in using a typed functional language for the development of a security application
}

\author{
Damien Doligez \\ Inria \\ damien.doligez@inria.fr
}

\author{
Christèle Faure \\ SafeRiver \\ christele.faure@safe-river.com \\ Manuel Maarek \\ SafeRiver \\ manuel .maarek@safe-river.com
}

\author{
Thérèse Hardin \\ UPMC \\ therese.hardin@upmc.fr
}

In this paper we present our experience in developing a security application using a typed functional language. We describe how the formal grounding of its semantic and compiler have allowed for a trustworthy development and have facilitated the fulfillment of the security specification.

\section{Introduction}

Developing an application with strong security requirements presupposes to identify the possible threats and to address all of them. Adverting or diverting the execution of an application is the goal of any attack which means that its targets span almost all the stages of development of the application and every aspect of its execution. On a first step in using a Formal Integrated Development Environment, we developed an industrial security application which was independently successfully assessed. This development was part of a study initiated and funded by the French Network and Information Security Agency (ANSSI ${ }^{1}$ ). The overall study was carried out by participants from industry (SafeRiver ${ }^{2}$, Normation ${ }^{3}$ and Oppide ${ }^{4}$ ) and academia (CEDRIC 5 and Inria ${ }^{6}$. The subject of this study was to determine what features of functional programming languages can help to prevent attacks and what are the ones which bring vulnerabilities. The results can be found on the ANSSI website 7 The development part of the study was led by SafeRiver and was informally and separately evaluated by an ANSSI team and an independent assessor. In this paper, we present this experience of development. It uses a typed functional language. We describe how the formal grounding of the semantics of this language and its compiler have allowed for a trustworthy development and have facilitated the fulfillment of the security specification. We did not address a formal description of security requirements nor mechanically-checked proofs of their fulfillments. But, some of the requirements have been reified onto type properties and thus automatically checked by the typing phase of the compilation process.

\footnotetext{
1 http://www.ssi.gouv.fr/

2 http://www.safe-river.com/

3 http://www.normation.com/

${ }^{4}$ http://www.oppida.fr/

5 http://cedric.cnam.fr/

6 http://www.inria.fr/

7 http://www.ssi.gouv.fr/fr/anssi/publications/publications-scientifiques/autres-publications/ lafosec-securite-et-langages-fonctionnels.html
}

Dubois C., Giannakopoulou D., Méry D. (Eds): Formal Integrated Development Environment 2014 (F-IDE 2014)

EPTCS 149, 2014, pp. 58-63, doi 10.4204/EPTCS.149.6 (c) D. Doligez, C. Faure, T. Hardin, M. Maarek

This work is licensed under the Creative Commons Attribution License. 


\section{Requirement specification}

The project goal was to develop a secure XML validator. XML is commonly used as a representation for data communicated by components of a system. The parser reading the data is therefore becoming the target of numerous attacks. There are two major sources of attacks: the data XML file and the parser itself. A maliciously forged XML file given as an entry to a system can give the whole system control to the attackers. Thus an XML validator is used to reject suspicious XML data entries, before transmitting the XML data to any critical parser of the system. The specification of the XML validator we developed is to comply to the most commonly used XSD constructions and to do so in a secure manner, that is, to resist to direct attacks.

\subsection{Functional requirements}

The system must be able to read an XSD format and to validate it. Then, if the XSD entry is acceptable, the tool has to generate an XML parser according to this XSD format. The central functional requirement is that the produced XML parser validates a file only if it is a well-formed XML file and if its content is valid according to the XSD format. The application has to comply with the $\mathrm{W} 3 \mathrm{C}$ recommendations on XML and XSD. Most of the XSD and XML constructions should be considered. Only those identified as "well-known as dangerous ones" by the ANSSI client must be rejected. For instance, the wild-card XSD constructs such as <xsd:any> were discarded as they are too permissive. We also forbid the use of numeric character references. They allow three representations for each standard characters (e.g., the s character could be represented in XML by its numeric character reference \&\#115; or \&\#x73;) which could confuse an XML parser.

\subsection{Security requirements}

We made a security analysis of the XML language, the XSD language and XML validations to define the security requirements the XML Validator has to fulfill.

Availability. A major requirement for a validation service is not to disrupt the function it is supposed to protect. A valid input for the service must not be rejected and must be treated in a reasonable time. A Denial of Service attack would make use of any input file pattern for which the validation process is inefficient. Moreover, erroneous invalidation of input files could lead to the whole system being moved to a degraded mode, and therefore not carrying out its duty.

Integrity and confidentiality. In addition to delivering a trustworthy validation result, the application has to guarantee the integrity and confidentiality of the data it manipulates. Moreover, the application's own integrity must be guaranteed in order to serve its security role. A code injection attack would use any potential flaw in the software that would permit to execute part of the input file content.

Auditability. A prevalent requirement is the ability to demonstrate the security of the application. For the application to be deployed it would need to be evaluated by an independent body and deemed to comply with the appropriate standard and assurance level: the Evaluation Assurance Level (EAL) of the Common Criteria 8 (ISO 15408) or the First level security certification for information technologies

\footnotetext{
${ }^{8}$ http://www . commoncriteriaportal.org/
} 
$(\mathrm{CSPN} 9)$ in the French context.

\section{Development environment for security}

The development environment used for the development of the application is OCaml 10 The OCaml language is strictly typed and functional. OCaml's standard development environment comprises a type checker, a native code compiler, and runtime system which are grounded on well studied formal foundations. The OCaml toolkit could be extended with various tools that are out of scope of this paper. We go into details of specific aspects of the OCaml language and its environment which played a crucial role in fulfilling the security requirements of the XML validator.

\subsection{Control flow analysis enabled by the functional approach}

Analysing the control flow of an application dealing with XSD and XML is important as these descriptive languages are complex. The application is required to provide a negative validation result when provided with a file that does not comply with either XML or the given XSD as it would be a security breach. It is also required not to give a negative validation result when provided with a valid file as it would result in opening the possibility of Denial of Service attack.

We designed the validation function to be purely functional. This is a very important point. First, whatever is the occurrence of a call to this function, its meaning is the same as it cannot depend on the memory state. It eases a lot the traceability of the computation of the result. Second, the formal studies of such pure typed functional functions mathematically demonstrate that, if the function can be typed, then its execution ends with a result of the attended type or loops for ever. This last case being eliminated by a simple study of recursive calls showing a decreasing measure, we can state that the XML validator supplies a validation result.

As OCaml is not purely functional, we identified the imperative constructions of the language and forbid their use within the code composing the validation function. More precisely, the following features were forbidden:

- The use of mutable variables as they make the control flow more complex to follow by proofreading,

- The use of exceptions for nominal computation as they disrupt the execution flow of a program,

- The use of non exhaustive pattern matching as it would introduce possible failures of the application.

To forbid such constructions, we have imposed some programming rules to follow. These rules are of a small number. They could be verified by proofreading of the code. Some rules could well be implemented as static verifications on the software code or on intermediate abstract representations provided by the compiler.

\subsection{Encapsulation as data protection}

To guarantee that neither the XML content to be validated nor the validated XSD grammar subjacent to the used XML validator are modified by the application, we needed to protect the data against alter-

9 http://www.ssi.gouv.fr/en/certification/first-level-security-certification-cspn/

10 http://caml.inria.fr/ 
ation. Values are, by default, immutable in OCaml. The memory initialisation and manipulations are automatically performed by the compiler and the Garbage Collector of the runtime system.

Thus there is no way of changing already acquired values if no mutable values are used. However, some harmful side effects are needed to input data. We therefore decided to restrict the usage of mutable values to isolated parts of the software and to justify this usage in the documentation. Then, we were faced with an OCaml feature: the string values are mutable in OCaml. However, the application makes intensive manipulations of strings and we needed to retain the efficiency of native string tools. Thus, to benefit from the native string library capabilities and in the meantime to forbid the mutation of these values, we have systematically used a version of string encapsulated in a module. The module uses internally the OCaml string library but does only provide manipulation functions that cannot alter the values themselves.

To enforce this use of an alternative version of the string, we have followed a number of coding rules which help to identify the use of mutable strings and which prevent the bypassing of modular encapsulation.

Another important gain in using OCaml is the fact that data and executable code are strictly separated in the native code produced by the compiler. To enforce such separation, we forbid the use of dynamic code loading and the use of the bytecode execution which are the only situations when data could be executed. As OCaml does not have pointers, the integrity of both data and executable code are guaranteed. This characteristic makes impossible any injection code attack.

\subsection{Types for traceability and prototyping}

During the validation of an XML file in accordance with an XSD grammar, the various constructions of the XSD and XML languages are explored. The validation process is decidable but the number of XML and XSD constructions, their combination and the number of validation rules specified in the W3C Recommendations increases the complexity of both the development and evaluation of the application. During the development of the XML validator, we heavily relied on the union types and record types of the OCaml language, not only to encode data but also to record the application of some $\mathrm{W} 3 \mathrm{C}$ rules. We can say that a large part of verifications of conformance to the $\mathrm{W} 3 \mathrm{C}$ recommendations was done through typing and pattern-matching. Indeed, the OCaml compiler systematically verifies that each operation applied on the values of these types by pattern matching covers every possible case, a point which improves the robustness of the application. To reinforce this robustness throughout the development, we forbade the use of catch-all patterns as they create a fragile matching. That is, in a pattern matching for a type composed of three constructs (e.g. type $t=A|B| C$ ), a catch-all pattern could be use to discriminate every other cases but the first one (e.g. let $f=$ function $A->\ldots \mid \ldots->\ldots$ ). In the event of the introduction of a forth construct later in the development, the catch-all pattern might become erroneous as nothing was known of this forth case at the time the pattern was written (e.g. if case $D$ is added, the typing of $f$ would still hold). Not using fragile patterns would force to make explicit the treatment of the additional case (e.g. a function defined with explicitly listing of all cases would have to be updated in order to type check, let $g=$ function $A->\ldots \mid$ B $\mid C->\ldots$ would require an additional case | $\mathrm{D}->$...).

The use of the OCaml types and the analysis performed by the type system has proven to be decisive to systematically cover the numerous cases of XML validation. It has also enabled the possibility of a reliable development by prototyping stages. For each new prototype, the types used were extended to take into account the additional functionality. For example, the first prototype version addressed only the simple cases of XML patterns. The next versions added new patterns and new rules of increasing 
complexity. Having just to extend some union data types and some functions defined by pattern-matching gave the insurance that the addition of new features was conservative over the already done development and was easy to manage as the simple addition of new cases. Moreover, the OCaml type system eased the development of new functionalities by pointing at every location in the code that needed an update.

\section{Industrial experience and evaluation}

The development was planned in several phases. Each phase releasing a new prototype with additional functionalities and enacting the possibility of testing these new functionalities. The assistance provided by OCaml type system ensured the reliability of the delivery of each prototype.

The application was informally evaluated by an independent expertise. The evaluation has identified a flaw (repaired in the next version) in the handling of command line arguments which is not due to the use of OCaml. The evaluation has not been able to identify any flaw related to its primary purpose as a validator. Nevertheless, the fact that OCaml applications have rarely been evaluated for security meant that there are no tools currently available for analysing its source code and compiled code. Commonly used binary code analysers are of little help for the auditor. This lacking might slow a formal evaluation.

\section{Conclusion}

The experience of developing an XML Validator with OCaml has demonstrated that it is a suitable environment for security developments. Moreover, the ability to monitor the development of the application with prototyping phases is of high industrial relevance. The formal grounding of the OCaml language and type system makes OCaml and its compiler a high level development environment. The tooling available is nevertheless limited, especially in an industrial context. Thus, it would be interesting to develop an IDE for OCaml, dedicated to critical software production. Some recommendations on this usage of OCaml are given in the study LAFOSEC, which precises what are the features of Ocaml which must be kept and what are the ones that must or can be dropped, what extensions and new libraries are needed. To reach the possibilities of a F-IDE, a logical component should be integrated to such an IDE. Focalize can be a very good model of this integration as it is based on the functional kernel of Ocaml. Its current compiler ensures a complete computational and logical treatment of the source development, which issues Ocaml and Coq codes, very close to each other and to the source code. It guarantees also a very large sharing of object codes and it provides automatic documentation and some testing possibilities. To summarize, developing this XML validator within a F-IDE à la Focalize should end with a product able to pass all the controls needed by an evaluation at the upper-rate level of security standards.

\section{References}

[1] ANSSI (2011): Analyse des langages OCaml, Scala et F\#. Étude de la sécurité intrinsèque des langages fonctionnels (LaFoSec), ANSSI. SafeRiver, CEDRIC and Normation. In French. Available at http://www.ssi.gouv.fr/fr/anssi/publications/publications-scientifiques/ autres-publications/lafosec-securite-et-langages-fonctionnels.html

[2] ANSSI (2011): Modèles d'exécution du langage OCaml. Étude de la sécurité intrinsèque des langages fonctionnels (LaFoSec), ANSSI. SafeRiver, CEDRIC and Normation. In French. Available at http://www.ssi.gouv.fr/fr/anssi/publications/publications-scientifiques/ autres-publications/lafosec-securite-et-langages-fonctionnels.html. 
[3] ANSSI (2011): Outils associés au langage OCaml. Étude de la sécurité intrinsèque des langages fonctionnels (LaFoSec), ANSSI. SafeRiver, CEDRIC and Normation. In French. Available at http://www.ssi.gouv.fr/fr/anssi/publications/publications-scientifiques/ autres-publications/lafosec-securite-et-langages-fonctionnels.html

[4] ANSSI (2011): Recommandations relatives à l'utilisation du langage OCaml et à l'installation et la configuration des outils associés. Étude de la sécurité intrinsèque des langages fonctionnels (LaFoSec), ANSSI. SafeRiver, CEDRIC and Normation. In French. Available at http://www.ssi. gouv.fr/fr/anssi/publications/publications-scientifiques/autres-publications/ lafosec-securite-et-langages-fonctionnels.html

[5] ANSSI (2011): État des lieux des langages fonctionnels. Étude de la sécurité intrinsèque des langages fonctionnels (LaFoSec), ANSSI. SafeRiver, CEDRIC and Normation. In French. Available at http://www.ssi.gouv.fr/fr/anssi/publications/publications-scientifiques/ autres-publications/lafosec-securite-et-langages-fonctionnels.html

[6] ANSSI (2012): XML Validator Generator XSVGen. Étude de la sécurité intrinsèque des langages fonctionnels (LaFoSec), première tranche conditionnelle (TC1), ANSSI. SafeRiver, CEDRIC and Oppida. Available at https://github.com/ANSSI-FR/xsvgen

[7] Philippe Ayrault, Matthieu Carlier, David Delahaye, Catherine Dubois, Damien Doligez, Lionel Habib, Thérèse Hardin, Mathieu Jaume, Charles Morisset, François Pessaux \& Pierre Weis (2008): Trusted Software within FoCaL. In: CE\&SAR, Rennes, France, pp. 142-157.

[8] Tim Bray, Jean Paoli, C. M. Sperberg-McQueen, Eve Maler, François Yergeau \& John Cowan (2006): Extensible Markup Language (XML) 1.1 (Second Edition). W3C Recommendation.

[9] Shudi Gao, C. M. Sperberg-McQueen \& Henry S. Thompson (2011): W3C XML Schema Definition Language (XSD) 1.1 Part 1: Structures. W3C Candidate Recommendation. Available at http://www .w3.org/ TR/2011/CR-xmlschema11-1-20110721/.

[10] Xavier Leroy, Damien Doligez, Alain Frisch, Jacques Garrigue, Didier Rémy \& Jérôme Vouillon (2010): The Objective Caml system release 3.12 - Documentation and user's manual. INRIA. Available at http: //caml.inria.fr/pub/docs/manual-ocaml/

[11] David Peterson, Shudi Gao, Ashok Malhotra, C. M. Sperberg-McQueen \& Henry S. Thompson (2011): W3C XML Schema Definition Language (XSD) 1.1 Part 2: Datatypes. W3C Candidate Recommendation. Available at http://www.w3.org/TR/2011/CR-xmlschema11-2-20110721/ 